\title{
Is sarcopenia a real risk factor for mortality in patients undergoing hemodialysis?
}

\author{
Eun Hui Bae
}

Department of Internal Medicine, Chonnam National University Medical School, Gwangju, Korea

Received: April 1, 2019

Accepted: April 8, 2019

\section{Correspondence to}

\section{Eun Hui Bae, M.D.}

Department of Internal Medicine, Chonnam National University Medical School, 42 Jebong-ro, Dong-gu, Gwangju 61469 , Korea

Tel: +82-62-220-6503

Fax: +82-62-225-8578

E-mail: baedak@hanmail.net https://orcid.org/0000-00031727-2822

\section{See Article on Page 599-607}

Sarcopenia is defined as a chronic condition characterized by slow and progressive decline in muscle mass and function [1]. Currently, owing to lack of unified tests and diagnostic criteria for uremic sarcopenia, the diagnosis of this condition is primarily based on diagnostic criteria established by the European Working Group on Sarcopenia in Older People [2]. Sarcopenia shows a high prevalence ( $14 \%$ to $63 \%$ ) in patients with end-stage renal disease [3-5]. The pathogenesis of sarcopenia in patients undergoing hemodialysis (HD) remains unclear and is attributed to multiple contributors such as increased levels of pro-inflammatory cytokines, insufficient protein intake, and insulin resistance [6].

In the latest issue of the Korean Journal of Internal Medicine, Kim et al. [7] have reported that sarcopenia was strongly associated with long-term mortality and cardiovascular events in patients undergoing HD. The study included 142 prevalent HD patients who were followed-up prospectively over 4.5 years. Low muscle mass measured using bioelectrical impedance analysis (BIA) was defined as a lean tissue in$\operatorname{dex}($ LTI $) \geq 2$ standard deviations below the normal sex-specific mean for young adults. Low muscle strength was defined as handgrip strength (HGS) < $30 \mathrm{~kg}$ in men and $<20 \mathrm{~kg}$ in women. Sarcopenia was diagnosed in patients with reduced LTI and HGS. Low LTI and HGS were independently associated with mortality. The association between sarcopenia and mortality has been studied in several settings, including in patients with chronic kidney disease [8]. Interestingly, muscle strength and muscle mass (the two components of sarcopenia), do not share the same clinical relevance [9]. In fact, muscle mass is not the only determinant of muscle strength. During the aging process, the decline in muscle strength is significantly more rapid than the loss of muscle mass [10]. In contrast to studies reporting findings in the general population, a recent large-scale study observed that sarcopenia (based on most standard definitions and estimation of low muscle mass, regardless of indexing method), was not significantly associated with mortality after adjusting for covariates [11]. Notably, slow gait speed and low muscle strength were significantly associated with mortality in patients undergoing maintenance HD [11]. Several studies have demonstrated a significant association between sarcopenia and high mortality in patients undergoing dialysis [12-14]. The discrepancy across studies with respect to the as- 
sociation between sarcopenia and mortality in patients undergoing HD indicates that the assessment of muscle strength and mass may provide prognostic information regarding survival in patients undergoing HD. Accurate measurement of muscle mass and quality is technically difficult. Historically, the calf circumference was used to determine muscle mass; however, it was shown to be a poor marker of muscle mass with limited individual prognostic relevance [15]. BIA is a relatively inexpensive, quick, and simple technique; however, the patient's hydration status, the machine and the equations used can affect the results [16]. Dual-energy X-ray absorptiometry (DEXA) is the most common imaging-based method used in research and in clinical practice because it is a simple and widely available test that uses minimal quantities of ionizing radiation and is also accurate and easily reproducible [17]. However, DEXA can over or underestimate muscle mass depending on whether the appendicular lean mass is used for measurement [18]. Computed tomography and DEXA may more objectively evaluate skeletal muscle index; however, these tests are expensive and not always widely available. Thus, they are not easily applicable in routine clinical practice.

In conclusion, to identify variables that best detect sarcopenia and predict outcomes, the best tools for measuring each variable including muscle mass and muscle quality need to be determined.

\section{Conflict of interest}

No potential conflict of interest relevant to this article was reported.

\section{REFERENCES}

1. Cao L, Morley JE. Sarcopenia is recognized as an independent condition by an International Classification of Disease, Tenth Revision, Clinical Modification (ICD-10CM) code. J Am Med Dir Assoc 2016;17:675-677.

2. Cruz-Jentoft AJ, Bahat G, Bauer J, et al. Sarcopenia: revised European consensus on definition and diagnosis. Age Ageing 2019;48:16-31.

3. Kim JK, Choi SR, Choi MJ, et al. Prevalence of and factors associated with sarcopenia in elderly patients with endstage renal disease. Clin Nutr 2014;33:64-68.

4. Lamarca F, Carrero JJ, Rodrigues JC, Bigogno FG, Fetter
RL, Avesani CM. Prevalence of sarcopenia in elderly maintenance hemodialysis patients: the impact of different diagnostic criteria. J Nutr Health Aging 2014;18:710-717.

5. Ren H, Gong D, Jia F, Xu B, Liu Z. Sarcopenia in patients undergoing maintenance hemodialysis: incidence rate, risk factors and its effect on survival risk. Ren Fail 2016;38:364-371.

6. Fahal IH. Uraemic sarcopenia: aetiology and implications. Nephrol Dial Transplant 2014;29:1655-1665.

7. Kim JK, Kim SG, Oh JE, et al. Impact of sarcopenia on long-term mortality and cardiovascular events in patients undergoing hemodialysis. Korean J Intern Med 2019;34:599607.

8. Pereira RA, Cordeiro AC, Avesani CM, et al. Sarcopenia in chronic kidney disease on conservative therapy: prevalence and association with mortality. Nephrol Dial Transplant 2015;30:1718-1725.

9. Isoyama N, Qureshi AR, Avesani CM, et al. Comparative associations of muscle mass and muscle strength with mortality in dialysis patients. Clin J Am Soc Nephrol 2014;9:1720-1728.

10. Goodpaster BH, Park SW, Harris TB, et al. The loss of skeletal muscle strength, mass, and quality in older adults: the health, aging and body composition study. J Gerontol A Biol Sci Med Sci 2006;61:1059-1064.

11. Kittiskulnam P, Chertow GM, Carrero JJ, Delgado C, Kaysen GA, Johansen KL. Sarcopenia and its individual criteria are associated, in part, with mortality among patients on hemodialysis. Kidney Int 2017;92:238-247.

12. Matos CM, Silva LF, Santana LD, et al. Handgrip strength at baseline and mortality risk in a cohort of women and men on hemodialysis: a 4-year study. J Ren Nutr 2014;24:157-162.

13. Vogt BP, Borges MCC, Goes CR, Caramori JCT. Handgrip strength is an independent predictor of all-cause mortality in maintenance dialysis patients. Clin Nutr 2016;35:1429-1433.

14. Yoda M, Inaba M, Okuno S, et al. Poor muscle quality as a predictor of high mortality independent of diabetes in hemodialysis patients. Biomed Pharmacother 2012;66:266-270.

15. Yang M, Hu X, Xie L, et al. Screening sarcopenia in community-dwelling older adults: SARC-F vs SARC-F Combined With Calf Circumference (SARC-CalF). J Am Med Dir Assoc 2018;19:277.e1-277.e8.

16. Maden-Wilkinson TM, Degens H, Jones DA, McPhee JS. 
Comparison of MRI and DXA to measure muscle size and age-related atrophy in thigh muscles. J Musculoskelet Neuronal Interact 2013;13:320-328.

17. Guglielmi G, Ponti F, Agostini M, Amadori M, Battista G, Bazzocchi A. The role of DXA in sarcopenia. Aging Clin
Exp Res 2016;28:1047-1060.

18. Di Sebastiano KM, Mourtzakis M. A critical evaluation of body composition modalities used to assess adipose and skeletal muscle tissue in cancer. Appl Physiol Nutr Metab 2012;37:811-821. 